\title{
Simulating Cooperative Road Hazard Warning Application From the Perspective of V2V
}

\author{
Guangmin LIANG $^{1}$ \\ 1 The School of Electronics and Communication Engineering, Shenzhen Polytechnic, Shenzhen \\ 518055, China
}

Keywords Cooperative road hazard warning, inter-vehicle communication, rear-end collision

\begin{abstract}
Cooperative Road Hazard Warning (C-RHW) is an event-driven and active road safety application and is performed through direct V2V/I2V communications. Whether or not a C-RHW application could work well is much dependent on the application-working pattern, the current spatial-temporal relation with the hazard area, and network performance. This paper proposes to analyze and simulate C-RHW applications through extensive simulation. We find that for the design of a feasible C-RHW application, the allowable minimum value of sending frequency should not be below $10 \mathrm{~Hz}$, and much attention should be paid to improve the transmission latency. The results are expected to guide the design and deployment of collision avoidance systems.
\end{abstract}

\section{Introduction}

The sustained development of automotive and traffic technologies facilitates the human life, but results in the unintended frequent accidents and congestions. The vehicular communication technology enables the surrounding traffic detection and provides a new feasible approach to reduce or even eliminate various collisions (e.g. vehicle-vehicle, vehicle-pedestrian, and vehicle-animal). Cooperative Road Hazard Warning (C-RHW) is an event-driven functionality to inform about a specific event or a specific condition for improving the road safety, e.g. stationary vehicle accident, and emergency electronic brake lights. C-RHW is not sent regularly but only when a special situation or event occurred. C-RHW is expected to create us with a safer and more efficient driving environment and numerous convenient services.

Over the past few years, ETSI and ISO are completing the standardization of reference communication architecture for cooperative intelligent transportation systems and propose many kinds of cooperative road hazard warning applications. Generally, the road hazards detected by drivers or on-board sensors would be reported to other vehicles through vehicular communication technologies e.g. 802.11p, so that other drivers approaching those hazardous areas can be notified about the risk in advance. The primary benefit of this concept is to increase the safety while the effectivity of such application is greatly determined by the good design of collision avoidance systems.

Generally, vehicles drive fast during a long-time journey at highway and thus drivers are difficult to always protect their vehicles from danger only by themselves, so once a collision occurs, it is likely to result in a rather serious consequence, and additionally the rear-end collision occupies most of them. It is necessary to pay much attention to design more friendly and intelligent collision avoidance systems. Most the previous work [1-5] focuses on the selection and control of the related hardware devices. Recently, through vehicle-to-vehicle communication, much information can be quickly spread among vehicles, e.g. location, velocity, travel time, etc. All information could be integrated by on-board systems, so drivers would learn the surrounding traffic in real time.

In this paper, we propose to analyze and simulate C-RHW applications through extensive simulation. We find that for the design of a feasible C-RHW application, the allowable minimum value of sending frequency should not be below $10 \mathrm{~Hz}$, and much attention should be paid to improve the transmission latency. Therefore, inspired by our simulation results, engineers and developers could deeply understand how to improve the success rate of C-RHW applications. 


\section{Problem Statement}

\subsection{ITS Station Reference Architecture Overview}

Designing the vehicular communication protocol should obey the rules specified in ITS station reference architecture jointly specially by ETSI TC ITS [6] and ISO TC204 [7]. As represented in Figure 1, the ITS station reference architecture is composed of four horizontal layers as an extension of the ISO 7-layer OSI model, and two cross-layer entities, i.e. Management and Security entities. The upper layers (above networking and transport) make use of the underlying communications facilities provided [8].

The applications layer provides ITS service. Three classes of applications have been distinguished at this level: road safety, traffic efficiency and other applications. Each application can be assigned to one of the three identified application classes. The primary objective of applications in the road safety class (active and passive) is the improvement of road safety, e.g. highway collision warning, hazardous location notification, and emergency electronic brake lights. The primary objective of applications in the traffic efficiency class is the improvement of traffic fluidity, e.g. in-vehicle signage, green-light optimal speed advisory, and limited access warning. Other applications in the co-operative local services and global internet services classes advertise and provide on-demand information to passing vehicles on either a commercial or non-commercial basis, e.g. vehicle software provisioning and update, fleet management, and local electronic commerce.

The facilities layer provides generic support facilities to applications, e.g. support of generating, structuring and formatting of application messages in conformity with available European standards, currently Co-operative Awareness Messages (CAMs) and Decentralized Environmental Notification Messages (DENMs) are being standardized.

CAMs [9] are periodically broadcasted by the facilities layer at a given frequency that is determined by the communication management entity considering the current experienced context and profile. However, the generation of CAMs should comply with the rules as follows. i) The maximum time internal between CAM generations: 1s. ii) Minimum time interval between CAM generations is $0.1 \mathrm{~s}$. iii) Generation of a CAM when absolute difference between current heading (towards North) and last CAM heading $>4^{\circ}$. iv) Generation of a CAM when distance between current position and last CAM position $>5 \mathrm{~m}$. v) Generation of a CAM when absolute difference between current speed and last CAM speed $>1 \mathrm{~m} / \mathrm{s}$; vi) The generation rules are checked every 100ms. CAM mainly employs broadcasting routing mechanisms.

DENMs [10] are triggered by the detection of some events. They are repeated as long as the event is perceived by the ITS station. Typically, DENMs are signaled using the broadcast capability. The DEN management module located in the facilities layer provides interfaces to the corresponding cooperative safety applications and other facilities in order to collect the necessary information for constructing and updating DENM. The ITS station receiving the DENM is able to provide an appropriate Human-Machine Interface (HMI) warning to the driver who could utilize this warning and then takes timely actions for avoiding accident. DENM prefers to be routed by geographical algorithms. 


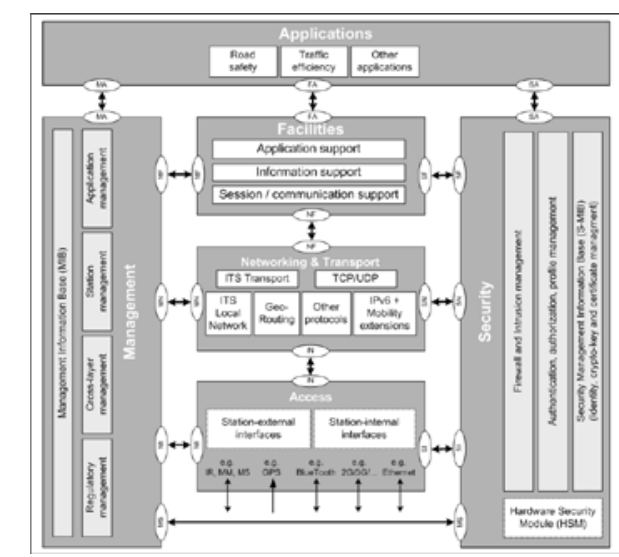

Fig.1 ETSI/ISO ITS Station Reference Architecture [6].

\subsection{Related Work}

In recent years, V2V-based safety driving assistance systems attract extensive attention from academic and industrial areas. Biswas et al. [11] give a collaborative highway anti-collision system by using DSRC (Dedicated short-range communications) to broadcast warning signals over a network. Nekovee [12] proposes a forwarding protocol between vehicles in order to defend against rear-end collisions on a highway. Taleb et al. [13] design an access control protocol to predict a danger and then improves the awareness of the anti-collision system in [11]. Tatchikou et al. [14] also propose a signal forwarding protocol for avoiding collisions. Most the previous work mainly focuses on how to design and implement an effective anti-collision communication protocol, but the detailed analysis on the happening probability of a rear-end collision is neglected. The discussions on connectivity between vehicles are also not enough. Ferreira et al. [15] make a comprehensive analysis on the connectivity problem in vehicular networks, but fail to model vehicular mobility in depth.

\section{Cooperative Road Hazard Warning Application}

Cooperative Road Hazard Warning (C-RHW) is an event-driven and active road safety application including numerous use cases, and is distributed among vehicle stations and roadside stations. The C-RHW application is performed through direct V2V /I2Vcommunications, and mainly utilizes DENMs to alert road users of the detected event. The general processing procedure of a C-RHW use case is as follows [10]. i) Upon detection of an event that corresponds to a C-RHW use case, the ITS station immediately broadcasts a DENM to other ITS stations located inside a geographical area which are concerned by the event. ii) The transmission of a DENM is repeated with a certain frequency. iii) This DENM broadcast persists until the detected event is disappeared. iv) The ITS stations receiving the DENMs process the information and decide to present appropriate warnings to drivers, as long as the DENM is relevant with the current ITS station. ETSI TC ITS has defined thirteen use cases of the C-RHW application. Table 1 provides examples of the triggering conditions of sending a DENM and its destination area [10]. 
Table 1 Triggering Conditions and Destination Area of Sending a DENM

\begin{tabular}{|c|c|c|}
\hline Use case & Triggering condition & Destination area \\
\hline $\begin{array}{l}\text { Emergency electronic brake } \\
\text { light }\end{array}$ & Hard breaking of a vehicle & $\begin{array}{l}\text { Rectangle covering road } \\
\text { topology }\end{array}$ \\
\hline $\begin{array}{l}\text { Wrong way driving } \\
\text { warning }\end{array}$ & $\begin{array}{l}\text { Detection of a wrong way driving by the } \\
\text { vehicle being in wrong driving direction }\end{array}$ & $\begin{array}{l}\text { Circle covering all } \\
\text { concerned road sections }\end{array}$ \\
\hline Stationary vehicle accident & e-Call triggering & $\begin{array}{l}\text { Rectangle covering road } \\
\text { topology }\end{array}$ \\
\hline $\begin{array}{l}\text { Stationary vehicle - vehicle } \\
\text { problem }\end{array}$ & $\begin{array}{l}\text { Detection of a vehicle breakdown or } \\
\text { stationary vehicle with activated warning }\end{array}$ & $\begin{array}{l}\text { Rectangle covering road } \\
\text { topology }\end{array}$ \\
\hline Traffic condition warning & Traffic jam detection & $\begin{array}{l}\text { Rectangle covering road } \\
\text { topology }\end{array}$ \\
\hline Signal violation warning & $\begin{array}{l}\text { Detection of a vehicle being violating a } \\
\text { signal }\end{array}$ & $\begin{array}{l}\text { Ellipse covering the } \\
\text { intersection area }\end{array}$ \\
\hline Road-work warning & $\begin{array}{l}\text { Signaled by a fix or moving roadside ITS } \\
\text { station }\end{array}$ & $\begin{array}{l}\text { Rectangle covering road } \\
\text { topology }\end{array}$ \\
\hline Collision risk warning & $\begin{array}{l}\text { Detection of a } \\
\text { rear-end/turning/crossing/merging } \\
\text { collision risk }\end{array}$ & $\begin{array}{l}\text { Ellipse covering the } \\
\text { intersection area }\end{array}$ \\
\hline Hazardous location & Detection of a hazardous location & $\begin{array}{l}\text { Circle covering all } \\
\text { concerned road sections }\end{array}$ \\
\hline Precipitation & $\begin{array}{l}\text { Detection of a heavy rain or snow } \\
\text { (activation of the windscreen wrappers) }\end{array}$ & $\begin{array}{l}\text { Circle covering all } \\
\text { concerned road sections }\end{array}$ \\
\hline Road adhesion & $\begin{array}{l}\text { Detection of a slippery road condition } \\
\text { (ESP activation) }\end{array}$ & $\begin{array}{l}\text { Circle covering all } \\
\text { concerned road sections }\end{array}$ \\
\hline Visibility & $\begin{array}{l}\text { Detection of a low visibility condition } \\
\text { (activation of some lights or anti-fog) }\end{array}$ & $\begin{array}{l}\text { Circle covering all } \\
\text { concerned road sections }\end{array}$ \\
\hline Wind & $\begin{array}{l}\text { Detection of a strong wind condition } \\
\text { (stability control of the vehicle) }\end{array}$ & $\begin{array}{l}\text { Circle covering all } \\
\text { concerned road sections }\end{array}$ \\
\hline
\end{tabular}

The most above C-RHW applications share some characteristics behind the triggering conditions and performance requirement on communication protocols. The vehicle ahead would start a C-RHW application and broadcast a DENM message periodically in certain frequency to the concerned area when detecting an exceptional event, e.g. a rear-end collision or a wild animal, so the latter vehicle could receive the DENM and provides the driver some suggestive coping strategy in accordance to the current traffic situation. Whether or not a C-RHW application could work well is much dependent on the application-working pattern (e.g. the sending frequency), the current spatial-temporal relation (e.g. the inter-distance, the initial driving velocity) with the hazard area (e.g. an accident vehicle or a wild animal), and network performance (e.g. transmission latency, packet loss probability). Considering the rear-end collisions contribute most percentage of crashes (both fatal and nonfatal) per year and without losing generality, we mainly focus on the rear-end collision, but the employed methodology and obtained results are also applied to other C-RHW applications.

C-RHW indeed supports the dissemination of the warning message among the vehicle station (V2V) and the roadside station (V2I). Compared to the 300m-communication range equipped in the vehicle station, the roadside station could cover $1000 \mathrm{~m}$ along the road due to the high installed position of the antenna, which undoubtedly contributes an improved effectivity and efficiency of detecting a warning signal. However, we aim to guide the design and deployment of collision avoidance systems, so the lower bound of the performance against which the successful avoidance of the chain rear-end collision could be tolerant is the key concern. The collision avoidance system 
inspired by the $\mathrm{V} 2 \mathrm{~V}$ is capable of behaving well in the better communication scenario where the $\mathrm{V} 2 \mathrm{I} / \mathrm{I} 2 \mathrm{~V}$ is available, but the converse case is not acceptable. So the value of the lower bound is decided by the solution of $\mathrm{V} 2 \mathrm{~V}$ rather than the V2I with respect to the transmission range and the availability of the RSU deployment. Therefore, only the V2V is focused in this paper.

Figure 2 illustrates a typical highway scenario in which three vehicles A, B, and C travel in the same direction one after one. At a given time t0, vehicle $\mathrm{B}$ collides with the ahead vehicle $\mathrm{A}$ and suffers from a rear-end collision, so vehicle B launches immediately a C-RHW application and periodically broadcasts DENM warning information to around vehicles through V2V communications (e.g. IEEE 802.11p) in order to avoid multi-driver pile-up. Wireless signal transmission is affected and limited by transmission distance and channel environment, resulting in a dramatic attenuation. So the latter vehicle $\mathrm{C}$ is possible to fail to receive warning signals. Hence vehicle B has to periodically broadcast warning signals in a certain frequency to backward vehicles. Vehicle $\mathrm{C}$ will continue moving forward in the original velocity until receiving warning signals. Once the warning signal is received, a HMI warning is provided to drivers or an avoidance action is triggered automatically. A general format of DENM and the stack workflow are also provided in Figure 2.

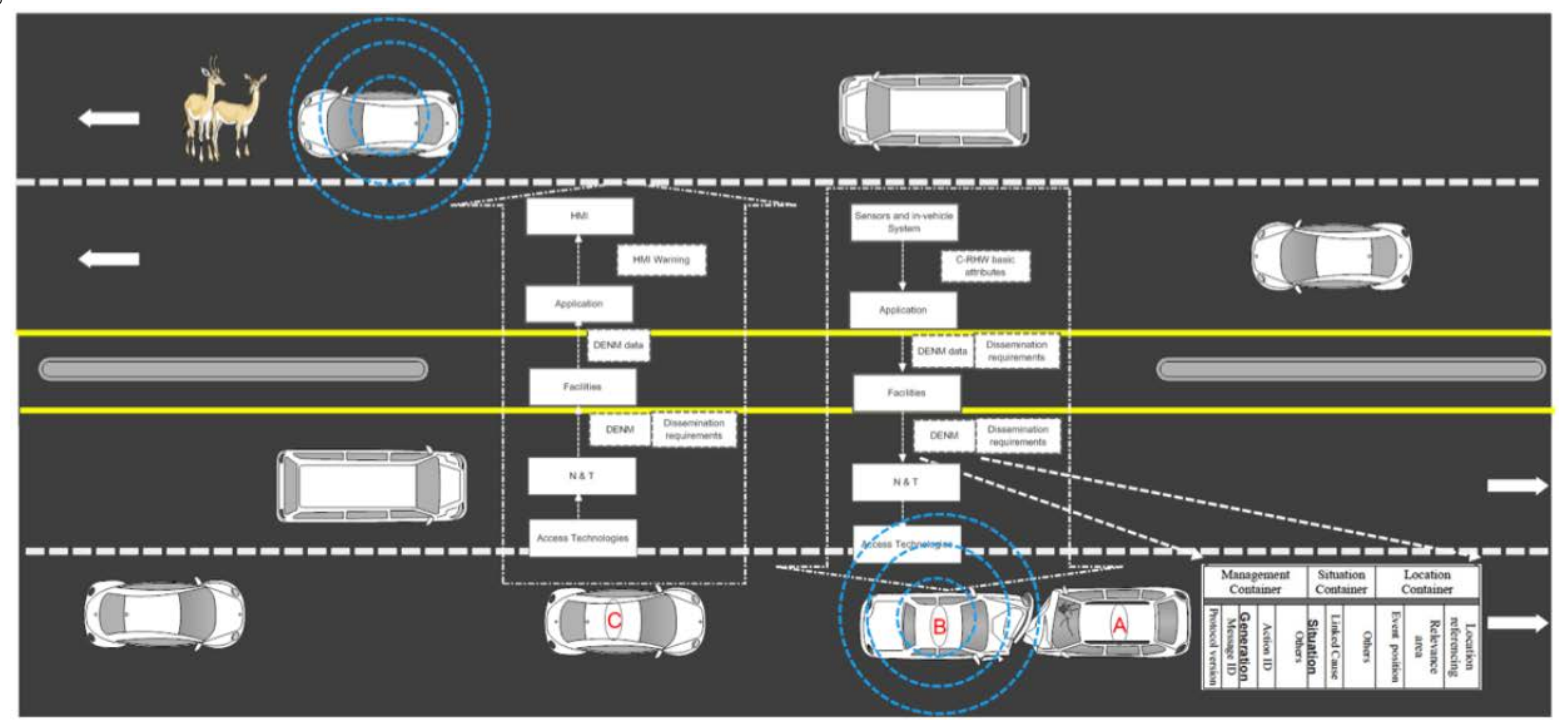

Fig.2 A demo for the typical C-RHW applications in a highway.

The latter vehicle $\mathrm{C}$ might have more than one choice to avoid a chain collision, such as immediate braking and lane switching. At one hand, the focused accident of the chain rear-end collision commonly occur at the highway, where the driving velocity is normally beyond $100 \mathrm{~km} / \mathrm{h}$, herein a sudden lane switching tends to cause sideslip or collision with other neighbor vehicles in the near lane. At the other hand, we aim to provide powerful driven towards a well design of V2V communication protocol for attaining the cooperative road hazard warning, so the C-RHW based ADAS enables a real-time feedback to the driver as a response to the sensed surrounding traffic, by which a safe enough inter-vehicle distance can be kept adaptively to the relative velocity during the whole journey. The outcome by the operation of lane-switch is not only upon the behavior of the current vehicle, but also relies on the reaction of the adjacent vehicle rather than the braking action where only the current vehicle plays a role. If the precaution of lane-switch is involved into calculating the safe inter-distance, the resulted value might be shortened compared to only adopting the braking under the precondition of empty at the proximity of the neighbor lane. However, the traffic is of high dynamic, so the traffic situation of the near lane happens to change quite frequently, resulting an oscillation of the required safe inter-distance between the current vehicle and the front vehicle. Therefore, as a precaution against the rear-end collision, such system should mainly depend on the braking action to make decision during a normal travel. Herein we focus on the case in which drivers are not permitted to occupy the neighbor lane and only to choose the emergent braking action for avoiding a collision. A driver should brake immediately in receiving a warning signal. If a vehicle could always keep a safe distance away from the accident vehicle ahead during the 
deceleration process, a chain rear-end collision is judged not to occur. (The safe distance affects the driving safety and false alarm rate. A small value decreases the safety otherwise the rate of false alarm rises. It is generally $2 \mathrm{~m}-5 \mathrm{~m}$ )

\section{Simulation}

We simulate the rear-end collision using Veins which is an open-source simulation framework and integrates both OMNeT++ and SUMO, the former is in charge of simulating communication and the latter controls mobility of vehicles. Veins supports the communication simulation based on 802.11p compatible with IEEE 1609.4 specification. The allowable maximum deceleration does not exceed $8.5 \mathrm{~m} / \mathrm{s} 2$, the driver's reaction time $\lambda 1=1$.3s and braking system's reaction time $\lambda 2=0.2 \mathrm{~s}$, as setup in theoretical analysis.

We investigate the critical values of velocity as well as inter-distance below which the rear-end collision exactly does not occur through varying transmission latency, packet loss probability, and sending frequency of DENM. During the process of data analysis, we could find the critical surface of diverse concerned metrics by using cubic polynomial interpolation. The critical surface of packet loss probability, sending frequency and transmission latency are provided in Figure 3(a), (b) and (c), respectively. For investigating which type of network metric could bring more affection on the effectiveness of C-RHW application, we normalize all the collected data of network metric including transmission latency, packet loss probability and sending frequency which determines the throughout. We combine and describe the normalized data in pairwise in one figure, and then drop shadow of their critical surface, as shown in Figure 4. The critical value of sending frequency experiences a fast uprising during climbing to the value of $10 \mathrm{hz}$, but after overpassing the critical point of $10 \mathrm{~Hz}$, the critical value of sending frequency behaves stably and fluctuates little. The curve of packet loss probability is nearly horizontal and brings little effect on this kind of application, i.e. C-RHW application. For design of a given C-RHW application, the allowable minimum value of sending frequency should not be below $10 \mathrm{hz}$. The critical value of transmission latency is apparently decreasing all along the whole observing window. Herein the transmission latency exerts the most important affection on the effectiveness of C-RHW application comparing other two network metrics, so one should pay much attention to improve transmission latency when designing the communicating protocols for a C-RHW application.
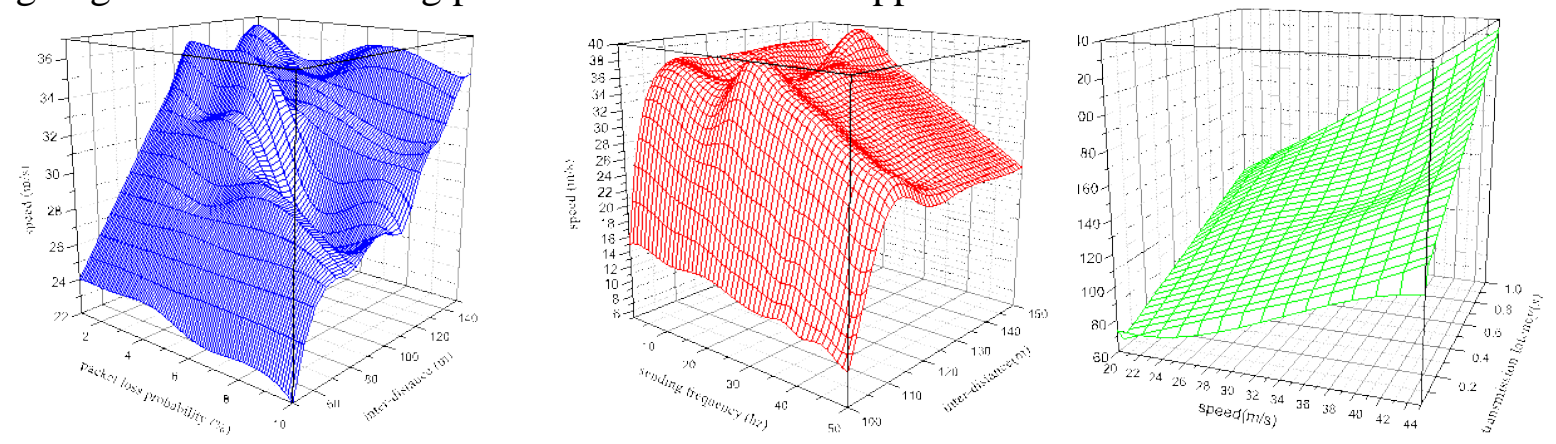

Fig.3 The critical surface of packet loss probability (a), sending frequency (b) and transmission latency(c). 

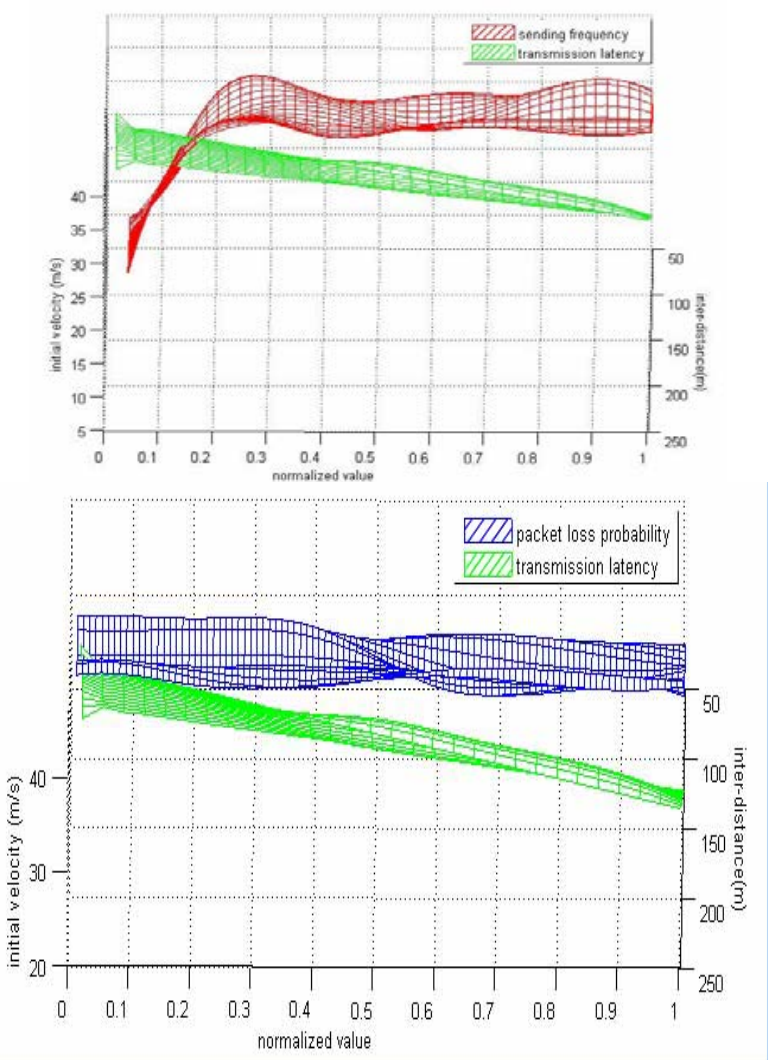

Fig.4 Critical surface shadow of combination between transmission latency and sending frequency (a) and combination between transmission latency and packet loss probability (b).

\section{Conclusions}

Most the previous studies about traffic safety pay much attention to sensors and image processing techniques, but these methods are dependent heavily on the surrounding environment, resulting in errors and cost. The emergence and development of vehicular communications inspire new solutions. This paper proposes to analyze and simulate C-RHW applications through extensive simulation. We find that for design of a C-RHW application, the allowable minimum value of sending frequency should not be below $10 \mathrm{~Hz}$, and much attention should be paid to improve transmission latency. The results could be used for guiding design and deployment of collision avoidance systems.

\section{Acknowledgements}

This work was supported by Shenzhen Strategic Emerging Industry Development Funds (JCYJ20130331151421558).

\section{References}

[1]. Chakravarthy A., Song K. Y., Feron E. (2004) A slowdown warning system for automobiles. IEEE International Conference on Systems, Man and Cybernetics, The Hague, Netherlands, 10-13 October 2004, pp. 3962-3969.

[2]. Bian M. Y., Li K. Q., Jin D. F., Lian X. M. (2005) A velocity control strategy for vehicular collision avoidance system. IEEE International Conference on Mechatronics and Automations, Ontario, Canada, 29 July - 1 August 2005, pp.93-95.

[3]. Barnes W. J., King T. I., Refai H. H., Fagan J. E. (2007) A wireless sensor network simulation for highway intersection collision prevention. IEEE Conference on Intelligent Transportation Systems, Seattle, September 30-October 3 2007, pp.94-97. 
[4]. Dagan E., Mano O., Stein G. P., Shashua A. (2004) Forward collision warning with a single camera. IEEE Intelligent Vehicles Symposium, Parma, Italy, 14-17 June 2004, pp. 37-42.

[5]. Cherng S., Fang C. Y., Chen C. P., Chen S. W. (2009) Critical Motion Detection of Nearby Moving Vehicles in a Vision-Based Driver-Assistance System. IEEE Transactions on Intelligent Transportation Systems 10(1), 70-82.

[6]. European Telecommunications Standards Institute (2010) ETSI EN 302 665: Intelligent Transport System (ITS); Communication Architecture, v1.1.1.

[7]. International Organization for Standardization (2010) ISO 21217: 2010: Intelligent Transport Systems - Communications Access for Land Mobiles (CALM) - Architecture.

[8]. European Telecommunications Standards Institute (2009) ETSI TR 102 638: Intelligent Transport System (ITS); Vehicular Communications; Basic Set of Applications; Definitions, v1.1.1.

[9]. European Telecommunications Standards Institute (2011) ETSI TS 102 637-2: Intelligent Transport System (ITS); Vehicular Communications; Basic Set of Applications; Part 2: Specification of Cooperative Awareness Basic Service, v1.2.1.

[10]. European Telecommunications Standards Institute (2010) ETSI TS 102 637-3: Intelligent Transport System (ITS); Vehicular Communications; Basic Set of Applications; Part 3: Specifications of Decentralized Environmental Notification Basic Service, v1.1.1.

[11]. Biswas S., Tatchikou R., Dion F. (2006) Vehicle-to-vehicle wireless communication protocols for enhancing highway traffic safety. IEEE Communications Magazine 44(1), 74-82.

[12]. Nekovee M. (2009) Quantifying Performance Requirements of Vehicle-to-Vehicle Communication Protocols for Rear-end Collision Avoidance. IEEE Vehicular Technology Conference. Barcelona, Spain, 26-29 April 2009, pp. 2084-2088.

[13]. Taleb T., Benslimane A., BenLetaief K. (2010) Toward an Effective Risk-Conscious and Collaborative Vehicular Collision Avoidance System. IEEE Transactions on Vehicular Technology 59(3), 1474-1486.

[14]. Tatchikou R., Biswas S., Dion F. (2005) Cooperative vehicle collision avoidance using inter-vehicle packet forwarding. IEEE Global Telecommunications Conference. Saint Louis, 28 November-2 December 2005, pp. 2762-2766.

[15]. Ferreira M., Conceicao H., Fernandes R., Tonguz O. K. (2009) Urban Connectivity Analysis of VANETs through Stereoscopic Aerial Photography. IEEE Vehicular Technology Conference. Barcelona, Spain, 26-29 April 2009, pp.863-865. 